INPLASY

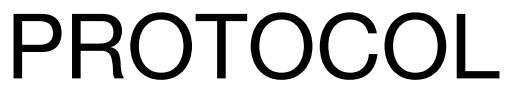

To cite: Deng et al. Efficacy and safety of Tian moxibustion in treating allergic rhinitis: a protocol for a systematic review and meta-analysis. Inplasy protocol 2020110058. doi:

10.37766/inplasy2020.11.0058

Received: 14 November 2020

Published: 14 November 2020

Corresponding author:

Gen Deng

1084053154@qq.com

Author Affiliation: Jiangxi University of

Traditional Chinese Medicine

Support: Jiangxi Education Department.

Review Stage at time of this submission: The review has not yet started.

Conflicts of interest: No conflict of interest.

\section{Efficacy and safety of Tian moxibustion in treating allergic rhinitis: a protocol for a systematic review and meta-analysis}

Deng, G1; Wan, Q2; Wang, J3; Ye, W4.

Review question / Objective: How about the efficacy and safety of Tian moxibustion in the treatment of allergic rhinitis. Condition being studied: Allergic rhinitis, abbreviated AR, modern medicine considers AR to be a chronic inflammatory reactive disease of the nasal mucosa mediated by exposure to allergens such as pollen and mites IgE. The prevalence of AR in Europe and the United States and China is 15\% 30\% and $4 \% \sim 38 \%$, respectively. About 600 million people worldwide suffer from the disease, and the incidence is gradually increasing. AR not only affects patients' daily life, sleep, work and study, but also brings huge economic burden to patients and society. Tian moxibustion is the use of irritating drugs on the skin applied to acupoints or affected areas, so that its local skin naturally filled Treatment of blood, flushing, or blister. Tian moxibustion therapy has a good effect on AR. However, there is still a lack of systematic evaluation on the efficacy and safety of Tian moxibustion therapy for AR in clinical practice. Therefore, the effectiveness and safety of Tian moxibustion in the treatment of AR will be systematically evaluated and meta-analyzed in this paper.

INPLASY registration number: This protocol was registered with the International Platform of Registered Systematic Review and Meta-Analysis Protocols (INPLASY) on 14 November 2020 and was last updated on 14 November 2020 (registration number INPLASY2020110058).

\section{INTRODUCTION}

Review question / Objective: How about the efficacy and safety of Tian moxibustion in the treatment of allergic rhinitis.

Rationale: A systematic review and metaanalysis of clinical randomized controlled studies on Tian moxibustion for allergic rhinitis following the rules of evidencebased medicine.

Condition being studied: Allergic rhinitis, abbreviated $A R$, modern medicine considers AR to be a chronic inflammatory reactive disease of the nasal mucosa mediated by exposure to allergens such as 
pollen and mites IgE. The prevalence of AR in Europe and the United States and China is $15 \% \sim 30 \%$ and $4 \% \sim 38 \%$, respectively. About 600 million people worldwide suffer from the disease, and the incidence is gradually increasing. AR not only affects patients' daily life, sleep, work and study, but also brings huge economic burden to patients and society. Tian moxibustion is the use of irritating drugs on the skin applied to acupoints or affected areas, so that its local skin naturally filled Treatment of blood, flushing, or blister. Tian moxibustion therapy has a good effect on AR. However, there is still a lack of systematic evaluation on the efficacy and safety of Tian moxibustion therapy for AR in clinical practice. Therefore, the effectiveness and safety of Tian moxibustion in the treatment of $A R$ will be systematically evaluated and metaanalyzed in this paper.

\section{METHODS}

Search strategy: Eight electronic databases will be searched, including PubMed, Embase, Web of Science, Cochrane Library, the China National Knowledge Infrastructure(CNKI), Chinese Science and Technology Periodical Database(VIP), Wanfang Database(WF), and Chinese Biomedical Literature Database(CBM). We will search above electronic databases from the beginning to November 2020, without any language restriction, but involving only the human subjects.

Participant or population: There are clear and recognized diagnostic criteria and efficacy criteria, and all patients are diagnosed as allergic rhinitis, regardless of gender, age and origin of the case.

Intervention: Tian moxibustion therapy, or mixed therapies based on Tian moxibustion will also be include.

Comparator: The control group will receive one of the following treatment methods: conventional pharmacological therapy, no treatment, and placebo.
Study designs to be included: Clinical randomized controlled trials (RCTs) containing Tian moxibustion for allergic rhinitis were included.

Eligibility criteria: Reported in chinese and english, and meet the "PICOS", will be considered for inclusion in this overview.

Information sources: Eight electronic databases will be searched, including PubMed, Embase, Web of Science, Cochrane Library, the China National Knowledge Infrastructure(CNKI), Chinese Science and Technology Periodical Database(VIP), Wanfang Database(WF), and Chinese Biomedical Literature Database(CBM). We will search above electronic databases from the beginning to November 2020, without any language restriction, but involving only the human subjects.

Main outcome(s): Clinical efficacy, including total effective rate or cure rate, and recurrence rate will be accepted as the primary outcomes.

Additional outcome(s): Rhinoconjunctivitis quality of life questionaire (RQLQ) score, symptom score (nasal congestion, snot, continuous sneezing) will be used as secondary outcomes.

Data management: The two researchers independently read the title and abstract of the literature they obtained, read the full text of the trials that might meet the inclusion criteria to determine whether the inclusion criteria were truly met, and discussed the conflicting literatures or let the third researcher decide whether to include them. Two researchers independently extracted data from the included studies, including study design, intervention measures and methods, measurement indicators, results, methodological contents such as hidden grouping and blind method, etc., and a third evaluator checked the consistency of the data. If the required information is incomplete, we will contact the original author for the required data. 
Quality assessment / Risk of bias analysis: The Cochrane Handbook of Systematic Review (5.1.0) RCT risk assessment tool was used to evaluate the risk of bias by two independent researchers, including: (1) The method of random sequence generation; (2) Allocation hiding; (3) Whether the subjects and the implementer of the treatment plan should be blinded; (4) Blind method shall be applied to evaluators; (5) Integrity of result data; (6) Selective reporting of results; (7) Other bias. According to the results of each study that meets the inclusion criteria, according to the above 7 items, objectively judge that each study is high-risk or low-risk or unclear (no relevant information or uncertainty of bias is mentioned in the literature) and explain the reasons. If there are any differences in the above quality evaluation and data extraction process, two people shall discuss and resolve the differences or consult the third reviewer to deal with the differences.

Strategy of data synthesis: The Cochrane Handbook of Systematic Review (5.1.0) RCT risk assessment tool was used to evaluate the risk of bias by two independent researchers, including: (1) The method of random sequence generation; (2) Allocation hiding; (3) Whether the subjects and the implementer of the treatment plan should be blinded; (4) Blind method shall be applied to evaluators; (5) Integrity of result data; (6) Selective reporting of results; (7) Other bias. According to the results of each study that meets the inclusion criteria, according to the above 7 items, objectively judge that each study is high-risk or lowrisk or unclear (no relevant information or uncertainty of bias is mentioned in the literature) and explain the reasons. If there are any differences in the above quality evaluation and data extraction process, two people shall discuss and resolve the differences or consult the third reviewer to deal with the differences.

Subgroup analysis: If significant heterogeneity is found in our systematic review and sufficient data is available, we will conduct a subgroup analysis based on moxibustion type, moxibustion time, treatment cycle, and outcome measurement methods in the experimental and control groups.

Sensibility analysis: When sufficient RCTs are available, we will conduct sensitivity analysis by excluding low-quality or highquality studies one by one according to methodological quality, sample size and missing data.

Language: No limitation of language.

Country(ies) involved: China.

Keywords: Tian moxibustion; moxibustion therapy; Allergic Rhinitis; Systematic review and meta-analysis.

Contributions of each author:

Author 1 - Gen Deng - The author drafted the manuscript.

Email: 1084053154@qq.com

Author 2 - Qun Wan - The author provided statistical expertise.

Email: 417503029@qq.com

Author 3 - Jinlong Wang - The author contributed to the development of the selection criteria, and the risk of bias assessment strategy.

Email: 1349556348@qq.com

Author 4 - Wenguo Ye - The author read, provided feedback and approved the final manuscript.

Email: 648957697@qq.com 